\title{
A Mini Review of Peer-to-Peer (P2P) for Vehicular Communication
}

\author{
Sumendra Yogarayan', Siti Fatimah Abdul Razak ${ }^{2}$, Afizan Azman³, and Mohd. Fikri Azli Abdullah \\ ${ }^{1,2,4}$ Faculty of Information Science and Technology, Multimedia University, Melaka, Malaysia \\ ${ }^{3}$ Kolej Universiti Islam Melaka, Melaka, Malaysia
}

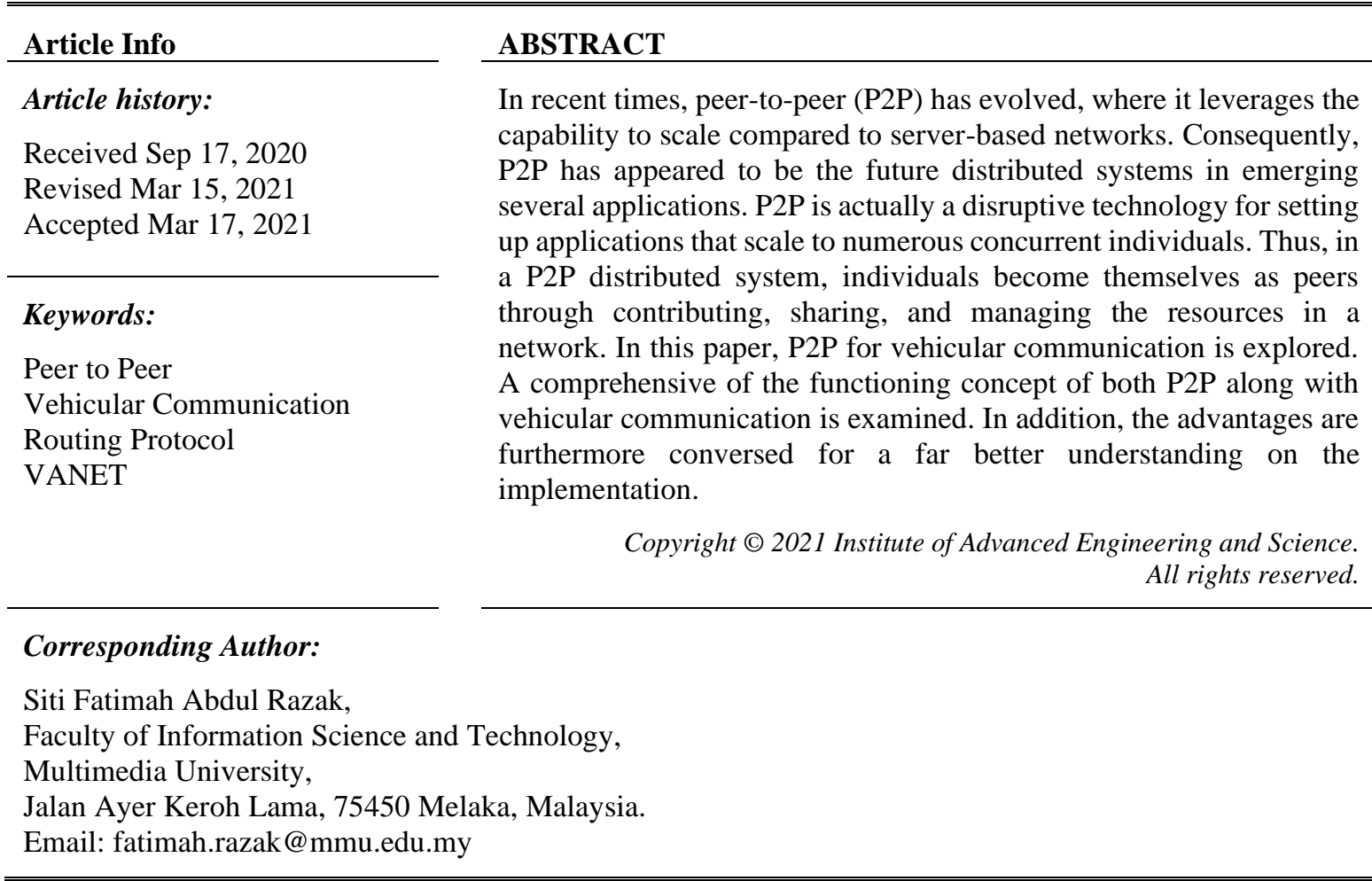

\section{INTRODUCTION}

Peer-to-peer (P2P) has ended up being exceptionally prominent in current times as a result of the great possibility to the high capability, effectiveness as well as fault resistance due to the fact that there is no centralized server and the network self-organizes itself [1]. Several study efforts in the field of P2P have actually concentrated towards purely performance problems such as scalability, reliable message transmission across the network, or accessibility to distributed sources [2]. In a P2P, peers link to each other directly for the exchange of information. File sharing is one particular example of this terminology, which has indeed been relatively effective and has attracted considerable interest [3]. These systems often involve numerous versatile peers associated with the sharing and cooperation procedure, without being conditional on the main authority. Besides, P2P is known as self-organized and highly decentralized. The inherent features of these kinds of systems have enabled the use of distributed P2P computing with study outlines [4].

$\mathrm{P} 2 \mathrm{P}$ is an excellent technique to develop trusted platforms for vehicular communication. The advancement of the network technology has actually provided using them in various areas. One of the emergent applications of them is the improvement of the vehicular communication in which the communications are between nearby vehicles, infrastructures, devices, or networks [5]. Vehicle communication has recently become to be a platform to maintain smart transportation system to enhance traffic safety. Vehicular communication is made up for a collection of communicating vehicle fitted with wireless devices that can communicate with each other without existing infrastructure [6]. One of the most crucial network technology offered lately for developing vehicular communication is the IEEE 802.11p standard known as Dedicated Short Range Communication (DSRC), however new requirements as 5th Generation (5G) is involving [7]. The exchange of information among the vehicles provides much chance for the development of advanced driver assistance systems. These systems will absolutely have the capacity to share and to gather real-time information 
concerning the number of various other vehicle, roadway traffic, and environmental conditions. Such information will certainly be managed and examined to sustain the driving experience by providing the drivers with necessary information. The road-constrained elements of these networks, the high mobility of the vehicles as well as the growth of roadside facilities make vehicular communication a stimulating and desirable research focus $[8,9]$.

\section{PEER TO PEER (P2P)}

$\mathrm{P} 2 \mathrm{P}$ is actually not a brand-new kind of modern technology. In reality, it is has actually been around considering that the advancement of the Internet where it originated with the e-mail process [10]. A P2P is a network of computers that each share an equal volume of responsibility in relation to process a data as shown in Figure 1. P2P differs from the client-server network, where some devices share the responsibility of processing as well as contributing information, while the various other devices take the data being actually refined by servers [11]. P2P networks was deployed for organization objectives back in the early 1980s. Just before P2P was put into usage, mainframes were actually used to hold documents on a central hard disk [12]. Visualizing the occurrences, it is inefficient and has produced a substantial mess of cables. Through technological advancement, computer systems can be designed which allow an individual to operate remotely without any necessity. In addition, any individual could share data and link printers to one another with the help of P2P computing [13].

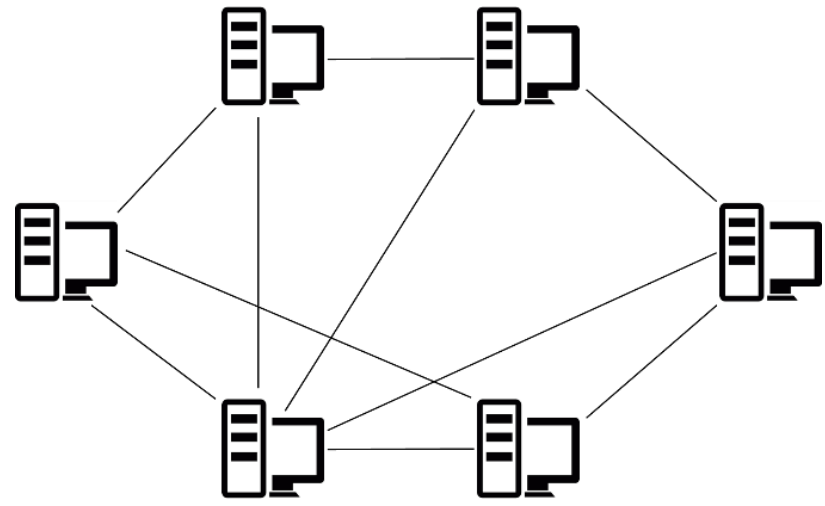

Figure 1. Peer to Peer Network

\subsection{How It Works}

Peer to peer (P2P) is actually protected by a distributed network of individuals. Generally, they possess no central system or server since each nodes keeps a duplicate of the documentations by functioning both as a client and as a server to other nodes [14]. Hence, each nodes can download and install and also put up files from different nodes or even upload information to them. This divides P2P networks from a network of data centers, through which client devices retrieve data from a central web server [15]. Apart from that, on $\mathrm{P} 2 \mathrm{P}$, the connected tools share files stored on respective disks. Utilizing software program applications designed to moderate the sharing of data, clients can request various other devices on the network to discover and download files [16]. When a client has downloaded a file, the client can act as a source of that data. However, when client are functioning as a server, they are the sources from which various other nodes can download the files. In practice, though, both functions can be carried out at the same time for instance, downloading data A, and uploading data B [17]. Since every node transmits, stores and receives files, P2P usually tend to become faster and as much more reliable as their base enlarges. Moreover, their distributed architecture makes P2P incredibly invulnerable to cyberattacks. Unlike standard models, P2P does not aspect of failure [18]. There are three types of $\mathrm{P} 2 \mathrm{P}$ overlay technologies that are called unstructured, structured and hybrid.

\section{a) Unstructured P2P}

Unstructured P2P networks do not present any specific organization of the nodes as the participants interact randomly with one another. These systems are considered robust versus high activity for instance, a number of nodes frequently joining and leaving the network [19]. Unstructured P2P networks are easier to build but might need greater CPU and memory use due to the fact that search queries are sent out to the greatest number of peers possible. This tends to flood the network with queries, particularly if a small number of nodes is providing the preferred content [20]. 
b) Structured $P 2 P$

In structured $\mathrm{P} 2 \mathrm{P}$, the network provides an organized architecture, allowing nodes to efficiently look for files, even if the content is not extensively readily available. In most cases, this is accomplished with hash functions that assist in database lookups [21]. Although structured networks might be more efficient, they tend to present greater levels of centralization and usually require greater setup and maintenance expenses. Apart from that, structured networks are less robust when faced with high rates of activity [22].

\section{c) Hybrid P2P}

Hybrid P2P networks integrate the traditional client-server model with some aspects of the peer-topeer architecture [23]. Compared to structured and unstructured, hybrid models tend to present improved overall performance. For instance, it may develop a main server that helps with the connection in between peers. Hybrid P2P usually combines each of these techniques to achieve considerable levels of performance and decentralization [24].

\subsection{Advantages}

a) Self-Organization

A P2P creates and organizes itself where each peer dynamically identifies and develops the network. P2P networks to distribute within the peer group according to their decisions and conditions [25]. For instance, a file sharing allows an individual to access a selection of the file. If a peer is strained and bad efficiency occurs, peers could shift on a different provider, which changes the network topology and effectively load balanced.

\section{b) Cost Saving}

A P2P network does not require a server because private terminals serve as a repository of information. Registered users may use their authorized or personal device to access any connected device [26]. Due to the fact that each workstation interacts with every other on the consigned network immediately, this can substantially minimize overhead expenses for big organizations.

\section{c) Less Failure}

A P2P network does not rely on a centralized system to distribute information. That implies each terminal can function independently of every other one. In the case, if among the computer system goes down for any reason, then the remainder of the network will not experience an interruption. The only limitation in this scenario is that the data on the server, which are already in significant issues, are not being obtained. [27].

\section{d) No Operating System}

A P2P network does not require a fully configured network operating system. This benefit is another outcome of the independent structure of this setup. Each computer system operates as its own server in a manner, keeping files for individual users while offering access to local files that are often under different profiles. In order to manage the network, it is possible to link each system to a Web or cloud based system. As such, individual users can stay productive as the companies can reduce technology and employment costs without affecting in adverse ways [28].

\subsection{Data Replication}

Decentralized data sharing is enabled by distributing data across all peers in a P2P system [29]. Therefore, P2P systems replicate data over a network in order to resolve this issue. In the case of a peer failure, several peers may find the same data to ensure availability. There are three techniques identified for the replication of data [30].

\section{a) Single-Master vs Multi-Master}

One instance of the server handles the master-site database that logs all changes in single-master. The master database is reproduced to any number of user databases. The user instances of server are optimized for read and write operations. Any compose operation received by a user is referred back to the master. The master regularly updates the user databases. However, a possible of bottleneck and point of failure may impose for a single-master replication. Besides, in multi-master, one or more instances of the server manages the master directory site database. Each master has a replication contract that specifies procedures for integrating the master databases. Each master duplicates to any number of user databases. As such, in the case if a master has a failure, another masters can take control of the replicas [31]. 


\section{b) Full vs Partial}

Full replication comprises storing a copy of each shared object on all participating sites. This helps with sustaining optimum availability as each site will certainly change any other site in case of a failure. Besides, also supports load balancing as all sites have the very same capacity. On the other hand, for partial replication, each site includes a copy of a shared object subset, such that objects replicated one site may vary from objects replicated on another site. This helps to reduce less storage space to be utilized and decreases the number of replica update messages, since updates are just propagated to concerned sites [32].

\section{c) Synchronous vs Asynchronous}

The synchronous replication updates propagation approaches that applies the changes to all replicas within the transaction context that initiates the updates. As such, all replicas have the very same condition as the transaction gives. This is achieved by implementing concurrency control mechanisms such as two-phase locking (2PL), timestamp-based algorithms, and two-phase dedicate procedure (2PC). In contrast, asynchronous replication updates approaches that will not change all the replicas within the range of the transaction that initiates the updates. In particular, the real transaction binds to all replicas quickly and then updates are propagated $[33,34]$.

\section{VEHICULAR COMMUNICATION}

Vehicular communication involves communication between vehicles, infrastructures, and external devices as shown in Figure 2. With advanced wireless communication technology, is it possible to share information through real-time communication platforms. This has carried to produce various applications to enhance safety and comfort of vehicles and interaction between passengers as well as the Internet. The standardization efforts on vehicular communication are underway to make smart transportation system safe, convenient and greener [35, 36].

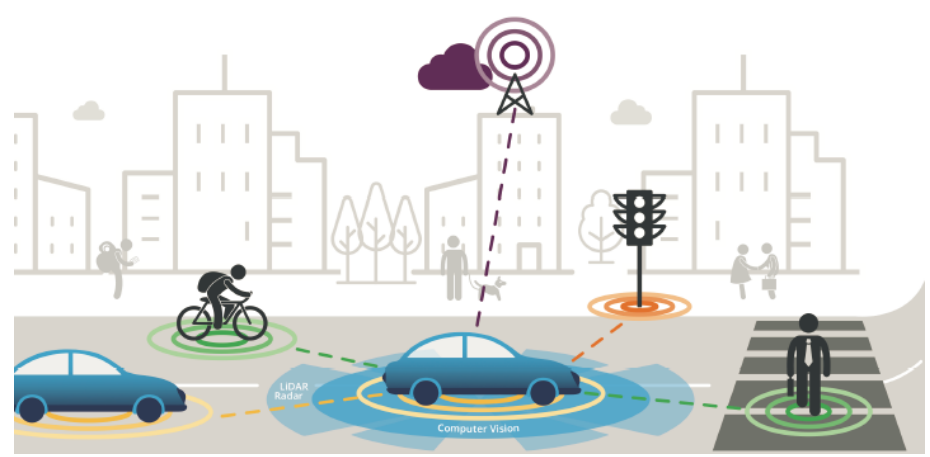

Figure 2. Connectivity of Vehicular Communication [37]

In vehicular communication, the potentials of sharing data such as position, speed, and location of the surroundings vehicles and infrastructures has provided a substantial result to reduce accidents. Vehicular communication could improve traffic congestion by alerting the driver of upcoming traffic jam location and recommending of alternative routes. This helps to ensure an eco-friendly driving by reducing the emission of a vehicle through smart transportation monitoring [38, 39].

\subsection{How It Works}

In general, vehicular communication operates with the use of Dedicated Short Range Communication (DSRC) protocol standard IEEE 802.11p. This protocol is a derivative of IEEE 802.11 (Wi-Fi) standard, which is specifically defined for high mobility nodes. This protocol standard uses a licensed spectrum 5.850 to 5.925 $\mathrm{GHz}$ band range. The functionality of this standard is that, it can operate in the circumstances of Non-Line of Sight (NLOS) difficulties and rough weather. Apart from that, in vehicular communication, there are different kinds of communications, such as, vehicle-to-vehicle (V2V), vehicle-to-infrastructure (V2I), vehicle-topedestrian (V2P), vehicle-to-network (V2N), vehicle-to-device (V2D) and vehicle-to-grid (V2G) shown in Figure 3 [40, 41, 42]. 


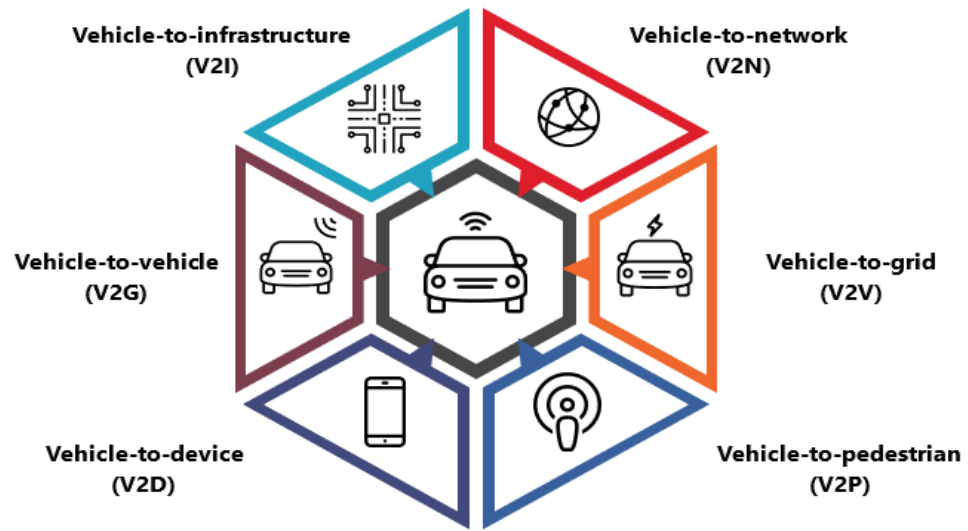

Figure 3. Types of Vehicular Communication [43]

\section{a) Vehicle to Vehicle}

Vehicle-to-vehicle (V2V) communications makes up a wireless network where vehicles send out messages per other with details. This information would include speed, location, as well as direction of traveling, braking, and loss of security of the particular car. For example, V2V cautions could pertain to the vehicle driver as a sharp, probably a traffic signal that flashes in the control panel, or an amber then red alert for escalating problems. It might suggest the direction of the danger [44, 45].

\section{b) Vehicle to Infrastructure}

Communication between vehicles to infrastructures (V2I) allows vehicles to communicate wirelessly with elements of bridge-related infrastructures such as road signage, traffic lights and lane markings. V2I modern technology assists in bi-directional wireless exchange of info between cars as well as the road facilities. The aim of this type of interaction is largely to make it possible for safety and security applications like staying clear of automobile accidents as a result of various reasons like roadway problems, climate condition, roadside building or malfunctioning traffic control. This technology alerts the motorist about accidents, traffic, sharp contours, and with suggested speeds [46, 47].

\section{c) Vehicle to Pedestrian}

The world health organization (WHO) have reports that each year almost 270,000 pedestrians pass away in road traffic accidents around the world. Vehicle-to-pedestrian (V2P) communication can change this terrible number for the better. Through V2P technology in smartphones as well as wearable gadgets, pedestrians can share information with vehicles. Together with sharing location information, pedestrian's gadgets can also signal drivers if, for example, they need more time to go across the road. This technology will protect a few of one of the most prone people when driving such as senior people and youngsters [48, 49].

\section{d) Vehicle to Network}

Vehicle-to-network (V2N) communication stands for the connection between the vehicles as well as a network driver supplying access to the vehicles, maybe on several frequencies. This communication can supply non-critical yet useful details such as on roadway closures or blockage and cloud-based services that enhance the experience of the residents, while also working as a hotspot for internet connectivity [50, 51].

\section{e) Vehicle to Device}

Vehicle-to-device (V2D) interaction is a system that connect vehicles to lots of outside obtaining devices but will be particularly advertised by two-wheeled travelers. It allows vehicles to interact wirelessly with the V2D tool such as bicycle. For instance, by sending out an alert of traffic ahead. Offered the reality that, in some countries, biking to job is the fastest-growing mode of transportation, boosting 60 percent in the past decade, V2D can potentially help protect against crashes [52, 53].

\section{f) Vehicle to Grid}

Vehicle-to-grid technology entails drawing extra power from the vehicle into the wise grid. V2G, which is also referred to as vehicle-grid integration (VGI), can aid the power grid supply electricity throughout optimal hours. It can likewise develop an extra source of power when weather-dependent renewable resource resources are not offered. As an example, a residence that makes use of solar energy cannot generate electricity at night, but an electrical vehicle might supply a second resource of power if required [54, 55]. 


\subsection{Advantages}

\section{a) Prevents Vehicle Collisions}

Vehicle collisions kill around thousands globally yearly, as well as the numbers keep rising each year. Safety has become a major concern and in spite of initiatives to raise understanding and enlighten on risk-free driving, the primary source of vehicle collisions continue to be by human careless. Vehicular communication can help to alleviate up to $70 \%$ to $80 \%$ of vehicle collisions including human negligent [56, 57].

\section{b) Improve of Traffic Congestion}

Vehicular communication could be used by law enforcement officials to keep a watchful eye and monitor traffic state with the use of real-time data transmission from vehicles to reduce traffic congestion. Vehicular communication could assist officials to route real-time traffic, track of vehicle locations, address speed restrictions, and reschedule traffic control measures. Drivers leveraging such systems can avoid the traffic congestion and maintain a safe distance with vehicles ahead $[58,59]$.

\section{c) Increase of Fuel Efficiency}

Vehicular communication allows heavy vehicle fleets to drive in close formation. For instance, a heavy vehicle in the front acts as a leader of the formation and the rest of the heavy vehicle will follow. The heavy vehicle on the platoon stay in constant formation and adjust to a constant stream of communication of their speed and position. Heavy vehicle platooning have shown that fuel consumption for the lead could reduce up to 5 percent, and for the following up to 10 percent $[60,61]$.

\section{d) Optimize Routes}

In sometime soon, as vehicles are completely commercialized with vehicle communication technology, each vehicle would be able to navigate with its best. The communication platform will provide accurate location, speed, and positioning information that will certainly help each vehicle maximize routes in real time [62].

\subsection{Routing Protocols}

Due to the movement of nodes, routing protocol for vehicular communication is important be considered, as vehicle move in a diverse speed and direction [63]. Another vital concern is to maintain a constant connectivity as some wireless networks may have problems like limited bandwidth, location-based network errors and channel variations. Routing protocols for vehicular communication have been widely researched and several protocols were suggested [64, 65]. Routing protocol can be briefly categorized into topology-based routing protocol, position-based routing protocol, cluster-based routing protocol, multicastbased routing protocol, geocast-based routing protocol and broadcast-based routing protocol [66].

\section{a) Topology}

In topology-based routing protocol, it uses link information that exists in the network to perform package forwarding. This protocol is classified into proactive, reactive, and hybrid. In proactive, the concept is actually to sustain a routing table for every node where route information is accessible immediately. Thus, this helps to reduce packet loss by updating the routing information constantly. Besides, in reactive, this routing protocol is referred to as on-demand-driven protocol. This protocol does not start route discovery by themselves, up until it has actually been made a demand to, when a source node requests for discovering a route. Nevertheless, this protocol utilizes a flooding mechanism for route discovery that initiates more routing overhead and suffers in the process with the initial route discovery. Apart from that, the hybrid protocol employs both the proactive and the reactive protocol capabilities and incorporates them to produce performance improvements. The network is divided into zones and utilizes several protocols in various zones from which some protocol in the zone is used and another in between $[67,68,69]$.

\section{b) Position}

Position-based routing protocol utilizes position data to identify where the specific location of the destination node and the neighbor node. Position-based routing does not require creating and maintaining routes in a network. It utilizes position information to offer more reliable and effective routing for certain applications and this information is normally acquired through location services and global positioning system (GPS). Therefore, to determine the destination address, the sender of the message uses a location service and include the message in the destination address. The location services can be categorized by the number of host nodes in which the position information can be gathered in different methods. The information can be collected from the direction and strength of the wireless signals received through a low-power GPS or a satellite transmitting signals to the GPS device [70, 71, 72]. 


\section{c) Cluster}

In cluster-based routing protocol, a group of nodes called clusters are partitioned in a network. This group of nodes have similar elements such as direction, location, and speed to form clusters. In every cluster, it has one cluster head, which are responsible for intra, and inter-controlling purposes. In intra-cluster, messages are initiated to member by cluster head whereas in inter-cluster communication between cluster heads are carried out by gateways. In this protocol, a node with high energy are selected for sending and processing data to other nodes. However, nodes with low energy are turned to account for sending and sensing data to cluster head $(\mathrm{CH})$. The attribute of this protocol adds to energy and scalability lifetime maximization $[73,74,75]$.

\section{d) Multicast}

In multicast-based routing protocol, messages are sent from a single sender to multiple destination in a specific geographical area. Multicast-based routing protocol is identified with some method such as flooding, proactive, and reactive. In flooding, the messages are transmitted across the network as a chain effect. The message to all neighbors other than the sender is sent by each node. Thus, the transmission of messages within the desired geographical area may be limited. Besides, in proactive, the routing information is maintained in the routing tables through a pre-computational routing. The routing tables are periodically maintained and updated whereby information across the network can be distributed. Along with that, in reactive, the routes are dependent on demand based upon a query-response process. When the query reaches the receiver, the connection between the sender and receiver is developed [76, 77, 78].

\section{e) Geocast}

A geocast-based routing protocol is a multicast subset with a multicast service that is achieved by the specification of the multicast group to be a specific geographical area. This in most cases contributes to nonoptimal protocols, particularly in ad hoc networks, in which the information of the geographical area is used to increase the efficiency of routing. On the other hand, if there is a geographical area that is addressed by a geocast protocol that includes nodes that should not accept a geocast message, then a geocast area may be refined by a multicast group. For instance, a multigroup can refine the geocast area if only vehicles and buses inside the geocast area are to be addressed instead of pedestrians. Nevertheless, geocast-based routing protocol is still the key mechanism for transmitting messages from a sender to a geographical area [79, 80, 81].

\section{f) Broadcast}

Broadcast-based routing protocol a basic method to broadcast messages from one node to all other nodes. The broadcast-based routing is a method that is one possible solution for data transmission in highmobility nodes. In a network, there is no maintenance required and information of individual nodes. Flooding is a mechanism used in a broadcast-based routing protocol. In the case if, a node rebroadcast a message although not knowing of the size of the message, a large number of redundant messages are transmitted into a channel that reduces the communication efficiency and wasting the bandwidth. Nevertheless, for data replication, it can be avoided by handling over an applicable relay for rebroadcasting the messages. Thus, the number data traffic in a network can be reduced significantly that leads to better utilization of bandwidth [82, $83,84]$.

\section{RELATED WORKS}

The development and incorporation of $\mathrm{P} 2 \mathrm{P}$ remains consistent as researchers are still active especially for vehicular communication.

Haq, A., et. al. (2020) presented two important contributions to the P2P communication based on data distribution algorithm. The author first suggested a game theoretical pricing model focused on the Rubinstein Bargaining. This model requires a relay node to price a service forwarder node depending on the remainder of the service level. Second, in order to enhance content distribution, the author has implemented P2P communication into the data-centric proposed algorithm. Message delivery ratio, average delivery delay of messages, overhead ratio, were the parameters used to measure the performance for the proposed algorithm. Therefore, the outcome have shown that, the proposed algorithm have reduced data delivery delays, have mitigate the selfishness in nodes, have a lesser overhead ratio and have increased the message distribution ratio [85].

Hassija, V., et. al. (2020) proposed a vehicle P2P network that would enable vehicles to securely communicate. There were two model proposed in this work. Firstly, an agreement in the vehicle network was achieved utilizing IOTA consensus mechanism. Secondly, an auction model was used to assign a maximum quantity of the necessary bandwidth. Nash bargaining algorithm has been applied to the existing algorithm in order to provide vehicles of optimum functionality. The relationship between vehicles that provide and consume offloading resources was modelled using a game-theoretical method. The outcomes of simulation 
showed that all vehicles on the network provide better Quality of Experience (QoE) and utility potential than their peers [86].

Hu, C. C. (2020) proposed a P2P timely distribution of information in VANET for sensitive deadline streaming. (TDDV). The mechanism is a different search method to gather the information needed and to seek segments in a particular province concurrently. The performance of the proposed mechanism were determined by using network simulator 2 (NS2). Simulation findings indicate that control bytes per second, admission rate, successful rate, single hop delay, route duration, and average route delay are higher than that of the existing mechanism. [87].

You, Z., et. al. (2019) delivered a unique software-defined network (SDN) directing scheme for P2P network. This scheme is designed for urban vehicle networks that can find a worldwide optimal path between source and destination. The algorithm described measures the global optimized routes for SDN and OpenFlow controllers vehicle communication and produces substantially lower end-of-end delay. This is a cross layer transmitting protocol in vehicle communication, where cross-layer metrics, including available bandwidth, link duration and forwarding probability will be used to determine the route from source to destination. As an effect, the routing decision based on route reliability reduces the likelihood of a disruption of the connection contributing to a lower rate of route interruption. The performance of this proposed scheme were analyzed at different speeds and density using a network simulator. The simulation settings that is configured were compared between SDGR with SDN, SVAO with SDN and AODV without SDN. The results have shown that the average end-to-end delay, throughput, overhead and packet drop ratio are superior to the other three protocols [88].

Yang, L., et. al. (2018) proposed a vehicle communication model based on P2P network that aims to resolve problem of blind spot and hot spot area. In this research, the base station and the roadside systems will no longer be required services as the vehicle can be made use as relays in the exchange of information. The relay nodes are selected based upon the degree distribution as well as the agreement process. With MATLAB simulation and exploration, the performance parameters of the traditional network of vehicles and the P2P vehicle network is compared. Thus, it is shown that the P2P vehicle network is cost-effective and capable in real-time [89].

Beak, B., et. al. (2018) developed a signal-priority control model, which forms the basis of multimodal intelligent traffic signal system (MMITSS). The MMITSS is a funded project by USDOT for cooperative transportation network, which emphasizes on the connected vehicle technology. The priority control model means that even a signal controller has a scalable possibility for vehicle priority policy. The priority control model reduces the number of failures and interruptions by reducing the undesirable impacts for vehicles. To evaluate the approach in a simulation experiment, two-VISSIM simulation networks were configured to assess the priority control of MMITSS, coordination, and fully operated control. The outcomes suggest that long-term peer-to-peer networking can enhance the reliability of transportation while reducing the negative impact on other mobility [90].

Malekian, R., et. al. (2018) defined a P2P data sharing system based on vehicular networks. The research aims to increase the situational awareness of driver and to reduce road accidents. The proposed system have incorporated several sensors to detect objects on the road and warn peer vehicles where alerts are send through roadside unit (RSU). The development consist of embedded system that is located in the vehicles and as well located in the roadside unit. The proposed system emphases on the distance sensor that conveys the distance and accuracy. The result proves that the proposed system is capable of communicating with vehicles and infrastructures to exchange information in order to avoid collisions and to facilitate safety driving [91].

Ferrag, M. A., et. al. (2017) introduced an effective secure routing scheme based on searchable encryption with vehicle proxy encryption, known as ESSPR, to ensure confidentiality message preservation in vehicular P2P network. The author has shown that ESSPR can be robust when processing packets, addressing interference, replay attacks, monitoring packets and wormhole attack. In terms of average fairness, delivery ratio, detection ratio and the average delay in VP2PSN, the effectiveness of ESSPR among malicious peers has shown that it is secure and efficient [92].

Meneguette, R. I., et. al. (2017) projected a method of mobile clouds based on vehicle communications supporting the exchange of information without the assistance of roadside infrastructures or external communication. In order to share and search available information, vehicles will have to cooperate with other vehicles. The simulation results show that the recommended approach presents a brief search time to inquire sources in one hop and to search for information in more than one jump. However, the approach provided approximately $95 \%$ with little overhead for high resource availability. The study has anticipate providing a peer-to-peer protocol that makes resources available for the vehicle and increases the amount of resources consumable in a vehicle cloud [93].

Eshteiwi, K., et. al. (2017) examined the effect of interference on the efficiency of vehicle to vehicle communications over individual and non-identically dispersed Nakagami-m fading channels. In this study, the 
communication of peer-to-peer vehicle to vehicle communication is derived, with the development of a closedform specification of the probability density function (PDF) and total signal-to-interference-plus-noise ratio (SINR). Besides, the representation of the moment generating function (MGF) is obtained from the previously mentioned PDF of the proposed method. An average bit error rate (ABER) closed form expression for vehicle to vehicle communication is also derived under the impacts of interference. In this study, the Monte Carlo simulation results has reflected the relation of the analytical expressions. Thus, this paper emphasizes on the impact of vehicle communication interference and presents essential mathematical expressions, such as PDF, SINR, MGF, and ABER [94]. Table 1 summarize previous studies.

Table 1. Summary of Previous Studies

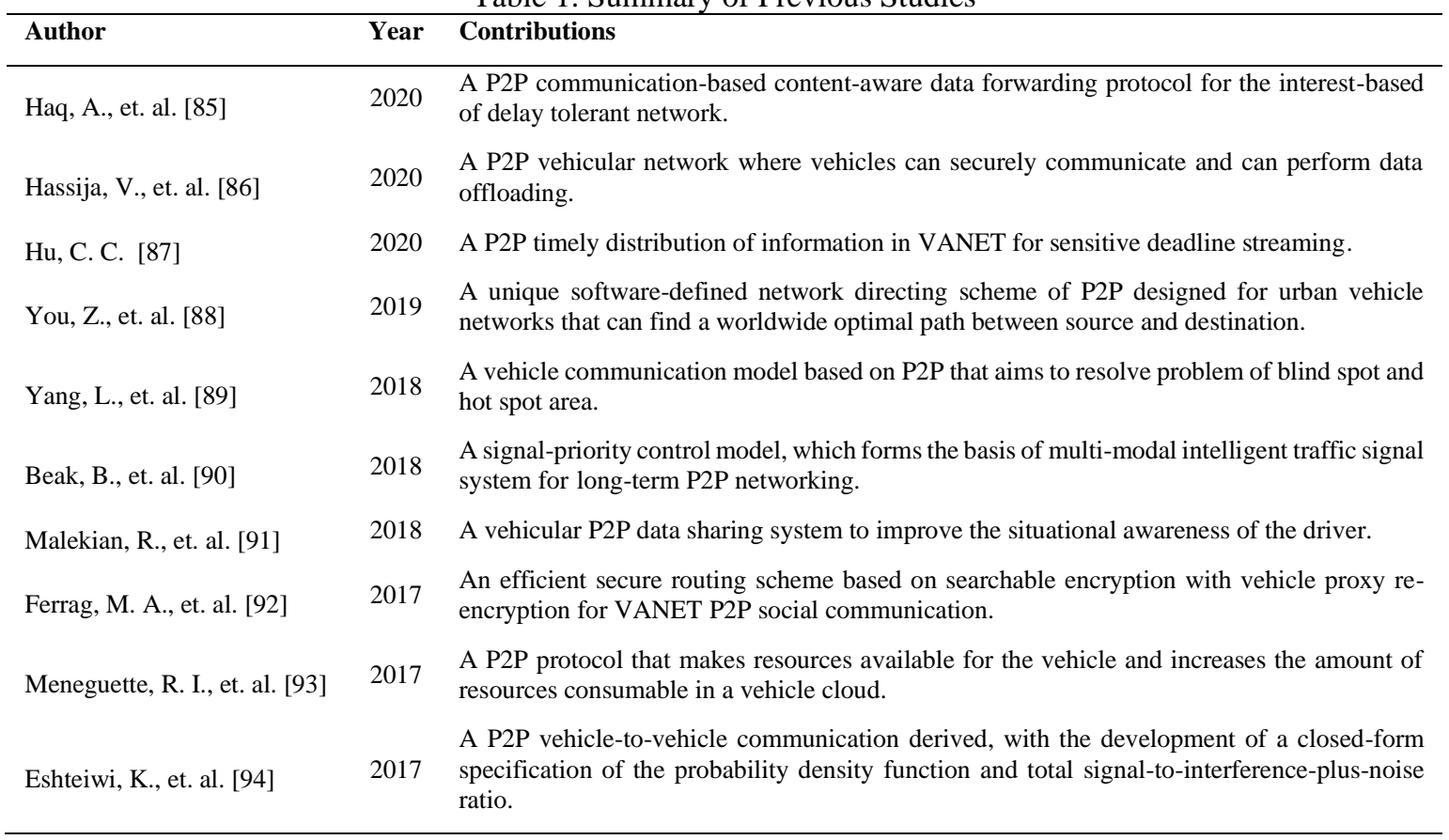

\section{CHALLENGES}

Although a peer-to-peer network is a great way to eliminate the server load, there are certain crucial concerns in order completely utilize for data exchange in vehicular communication environment. Most of the compact's difficulties are regarding security, performance, and regulation.

\section{a) Security}

A number of security aspects require to be addressed before undertaking a $\mathrm{P} 2 \mathrm{P}$ network for the vehicular communication. Many P2P systems make use of insecure protocols for communication as well as among numerous obtaining entailed peers. As soon as peers are able to obtain the information, it is almost impossible to sustain it. However, it is possible to protect the authenticity of the data with hash values and digital condensations, it may not be feasible to control replicating or removal of a data. For an example, a driver psychological profiling, it relies on precise and reliable data. As such, applications that generally involve offshelf devices that could be used as a connection to or from the monitoring data center with communication devices such as mobile phones. Thus, these data are necessary to be secured with the reality of non-replication or removal.

\section{b) Performance}

Attaining good performance is not trivial in $\mathrm{P} 2 \mathrm{P}$ systems, although it is a significant goal. The main paces in improving performance are distributing aggregating resources and processing load, such as storage and required processing power for peers. As a measure, some data processing in vehicles may be required because of the urgency or lack of connection to the base. This challenge covers, for example, the recognition and detection of fatigue detection using a combination of machine learning techniques and signal processing, which can be carried out on embedded systems. Such information is important because safety can be heavily dependent by both the driver and vehicle. 


\section{c) Regulation}

In a peer-to-peer network, the broader the network becomes the more complicated it becomes to manage and support and less scalable. The extent to which infrastructure deployment will shift the traditional vehicle regulation division, with the federal government being responsible for vehicle safety and the states concerned for the drivers aspects, such as licensing and registration, as driver and vehicle roles merge simultaneously. The national highway traffic security administration (NHTSA) survey has found that $94 \%$ of collisions are caused by human mistakes. Consequently, many assume that the advent of the intelligent transport system (ITS) is a crucial aspect of vehicular communication. As vehicular communication technology evolves, it is necessary for the government and local municipals to define the possible impacts of vehicles on roads.

\section{CONCLUSION AND FUTURE WORK}

The inevitable development and expansion of computers in decentralized information technology is $\mathrm{P} 2 \mathrm{P}$. Though many did not use the ability thoroughly, $\mathrm{P} 2 \mathrm{P}$ operating in vehicular communication mode may be a feasible solution for future transportation systems to be operated. P2P is a very effective method for developing efficient VANET platforms. In several specific ways the improvement of network infrastructure have made it possible to leverage them. The implementation of the VANET in which communication is between the V2V, V2I and V2G is among the evolving applications. Thus, this paper addresses P2P for vehicle communication. A detailed study of the $\mathrm{P} 2 \mathrm{P}$ and vehicle communication functioning concept is described. Therefore, the strengths are addressed in order to fully grasp the deployment. For future developments, the improvement in existing frameworks may provide new capabilities for vehicle communications. Therefore, the efficiency in a high mobility scenario may be assessed using these capabilities.

\section{ACKNOWLEDGMENTS}

This research is partially supported by FRGS/1/2019/TK08/MMU/03/2 grant. The authors fully acknowledged Ministry of Higher Education (MOHE) and Multimedia University for the approved fund, which makes this important research viable and effective. The authors gratefully acknowledge the use of service and facilities of the Connected Car Services Research Group, Centre of Intelligent Cloud Computing at Multimedia University. The authors would also like to thank the anonymous reviewers for their constructive comments.

\section{REFERENCES}

[1] Malatras, A.: State-of-the-art survey on P2P overlay networks in pervasive computing environments. Journal of Network and Computer Applications. 55, 1-23 (2015)

[2] Nobre, J. C., Melchiors, C., Marquezan, C. C., Tarouco, L. M. R., \& Granville, L. Z.: A survey on the use of P2P technology for network management. Journal of Network and Systems Management. 26(1), 189-221 (2018)

[3] Lu, K., Wang, J., \& Li, M.: An Eigentrust dynamic evolutionary model in P2P file-sharing systems. Peer-to-Peer Networking and Applications. 9(3), 599-612 (2016)

[4] Zhao, H., Ge, Y., Liu, Q., Wang, G., Chen, E., \& Zhang, H.: P2p lending survey: platforms, recent advances and prospects. ACM Transactions on Intelligent Systems and Technology (TIST). 8(6), 1-28 (2017)

[5] Aliyu, A., Abdullah, A. H., Kaiwartya, O., Cao, Y., Lloret, J., Aslam, N., \& Joda, U. M.: Towards video streaming in IoT Environments: Vehicular communication perspective. Computer Communications. 118, 93-119 (2018)

[6] Peng, H., Liang, L., Shen, X., \& Li, G. Y.: Vehicular communications: A network layer perspective. IEEE Transactions on Vehicular Technology. 68(2), 1064-1078 (2018)

[7] Filippi, A., Moerman, K., Martinez, V., Turley, A., Haran, O., \& Toledano, R.: IEEE802. 11p ahead of LTE-V2V for safety applications. Autotalks NXP. (2017)

[8] Collet, C., \& Musicant, O.: Associating vehicles automation with drivers functional state assessment systems: a challenge for road safety in the future. Frontiers in human neuroscience. 13, 131 (2019)

[9] Khan, M. Q., \& Lee, S.: A comprehensive survey of driving monitoring and assistance systems. Sensors. 19(11), 2574 (2019)

[10] Shiau, S. J., Huang, Y. C., Yen, C. H., Tsai, Y. C., Sun, C. K., Juang, J. N., ... \& Huang, S. K.: A novel massive deployment solution based on the peer-to-peer protocol. Applied Sciences. 9(2), 296 (2019)

[11] van Steen, M., \& Tanenbaum, A. S.: A brief introduction to distributed systems. Computing. 98(10), 967-1009 (2016)

[12] Anton, B., \& Norbert, A. Peer to peer system deployment. Acta Electrotech. Inform. 16, 11-14 (2016)

[13] Le, M., Clyde, S., \& Kwon, Y. W.: Enabling multi-hop remote method invocation in device-to-device networks. Human-centric Computing and Information Sciences. 9(1), 1-22 (2019)

[14] Wang, J., \& Liu, J.: The comparison of distributed P2P trust models based on quantitative parameters in the file downloading scenarios. Journal of Electrical and Computer Engineering. (2016)

[15] Orta-Cruz, C. A., \& de Asís López-Fuentes, F.: A Collaborative Platform for Secure Video Distribution. In 2018 IEEE 9th Annual Information Technology, Electronics and Mobile Communication Conference (IEMCON). 519-524 (2018) 
[16] Kleppmann, M., Wiggins, A., van Hardenberg, P., \& McGranaghan, M.: Local-first software: you own your data, in spite of the cloud. In Proceedings of the 2019 ACM SIGPLAN International Symposium on New Ideas, New Paradigms, and Reflections on Programming and Software. 154-178 (2019)

[17] Praveen, A. D., Kumar, K. D., Durai, M., \& Mary, A.: Collective Compress Support for Confidential Improvement on P2P Communications. (2018)

[18] Nicolini, A. L., Lorenzetti, C. M., Maguitman, A. G., \& Chesnevar, C. I.: Intelligent query processing in P2P networks: semantic issues and routing algorithms. Computer Science and Information Systems. 16(2), 409-442 (2019)

[19] Jogunola, O., Ikpehai, A., Anoh, K., Adebisi, B., Hammoudeh, M., Gacanin, H., \& Harris, G.: Comparative analysis of P2P architectures for energy trading and sharing. Energies. 11(1), 62 (2018)

[20] Ed-daoui13, I., El Hami, A., Itmi, M., Hmina, N., \& Mazri, T.: Unstructured peer-to-peer systems: towards swift routing. International Journal of Engineering \& Technology. 7(2.3), 33-36 (2018)

[21] Yeferny, T., Hamad, S., \& Yahia, S. B.: Query Learning-Based Scheme for Pertinent Resource Lookup in Mobile P2P Networks. IEEE Access. 7, 49059-49068 (2019)

[22] Shah, N., Ahmad, A., Nazir, B., \& Qian, D.: A cross-layer approach for partition detection at overlay layer for structured P2P in MANETs. Peer-to-Peer Networking and Applications. 9(2), 356-371 (2016)

[23] Duan, Z., Tian, C., Zhou, M., Wang, X., Zhang, N., Du, H., \& Wang, L.: Two-layer hybrid peer-to-peer networks. Peer-to-Peer Networking and Applications. 10(6), 1304-1322 (2017)

[24] Ali, S., Banerjea, S., Pandey, M., \& Tyagi, N.: Towards DHT-Based P2P Resource Sharing Over Hybrid Infrastructure of Wireless Mesh Network and Mobile. Computing and Network Sustainability: Proceedings of IRSCNS 2018. 75, 147 (2019)

[25] Tracey, D., \& Sreenan, C.: How to see through the Fog? Using Peer to Peer (P2P) for the Internet of Things. In 2019 IEEE 5th World Forum on Internet of Things (WF-IoT). 47-52 (2019)

[26] Ravichandran, C. G., \& Xavier, J. L.: A survey of data sharing and security issues in P2P networks. Advances in Natural and Applied Sciences. 11(7), 329-335 (2017)

[27] Kothari, R., Jakheliya, B., \& Sawant, V.: A Distributed Peer-To-Peer Storage Network. In 2019 International Conference on Smart Systems and Inventive Technology (ICSSIT). 576-582 (2019)

[28] Gattermayer, J., \& Tvrdik, P.: Using bootstraping principles of contemporary P2P file-sharing protocols in large-scale grid computing systems. In 2017 25th Euromicro International Conference on Parallel, Distributed and Network-based Processing (PDP). 214-218 (2017)

[29] Gallay, O., Korpela, K., Tapio, N., \& Nurminen, J. K.: A peer-to-peer platform for decentralized logistics. In Proceedings of the Hamburg International Conference of Logistics (HICL). 19-34 (2017)

[30] Spaho, E., Barolli, A., Xhafa, F., \& Barolli, L.: P2P data replication: Techniques and applications. In Modeling and Processing for Next-Generation Big-Data Technologies. 145-166 (2015)

[31] Gattermayer, J., \& Tvrdik, P.: Blockchain-based multi-level scoring system for P2P clusters. In 2017 46th International Conference on Parallel Processing Workshops (ICPPW). 301-308 (2017)

[32] Spaho, E., Barolli, L., \& Xhafa, F.: Data replication strategies in P2P systems: A survey. In 2014 17th International Conference on Network-Based Information Systems. 302-309 (2014)

[33] Ezéchiel, K. K., Kant, S., \& Agarwal, R.: Mediation of Lazy Update Propagation in a Replicated Database over a Decentralized P2P Architecture. Global Journal of Computer Science and Technology. (2019)

[34] Vashisht, P., Sharma, A., \& Kumar, R.: Strategies for replica consistency in data grid-a comprehensive survey. Concurrency and Computation: Practice and Experience. 29(4) (2017)

[35] Arena, F., \& Pau, G.: An overview of vehicular communications. Future Internet. 11(2), 27 (2019)

[36] Lozano Domínguez, J. M., \& Mateo Sanguino, T. J.: Review on V2X, I2X, and P2X Communications and Their Applications: A Comprehensive Analysis over Time. Sensors. 19(12), 2756 (2019)

[37] General, J. S.: Connectivity Standards in the Automotive Industry. 5GAA. 1-41 (2019)

[38] Chang, X., Li, H., Rong, J., Huang, Z., Chen, X., \& Zhang, Y.: Effects of on-board unit on driving behavior in connected vehicle traffic flow. Journal of Advanced Transportation. (2019)

[39] Balasubramaniam, A., Paul, A., Hong, W. H., Seo, H., \& Kim, J. H.: Comparative analysis of intelligent transportation systems for sustainable environment in smart cities. Sustainability. 9(7), 1120 (2017)

[40] Festag, A.: Standards for vehicular communication-from IEEE 802.11 p to 5G. e \& i Elektrotechnik und Informationstechnik. 132(7), 409-416 (2015)

[41] Azman, A., Yogarayan, S., Jian, S. L. W., Razak, S. F. A., Raman, K. J., Abdullah, M. F. A., ... \& Muthu, K. S.: A Study of Wireless Communication Technologies for Vehicular Communication. In International Conference on Information Science and Applications. 3-13 (2018)

[42] Tokody, D., Mezei, I. J., \& Schuster, G.: An overview of autonomous intelligent vehicle systems. In Vehicle and Automotive Engineering. 287-307 (2017)

[43] Martin, A., \& Ivanov, I.: Tapping into the Connected Cars Market: What You Need to Know | Access Partnership. https://www.accesspartnership.com/tapping-into-the-connected-cars-market-what-you-need-to-know/

(2018). Accessed 16 April 2020

[44] Zeadally, S., Guerrero, J., \& Contreras, J.: A tutorial survey on vehicle-to-vehicle communications. Telecommunication Systems. 73(3), 469-489 (2020)

[45] Feroz, A.: Vehicle to vehicle communication for collision avoidance. International Journal of Emerging Technology and Innovative Engineering. 5(7) (2019)

[46] Akabane, A. T., Immich, R., Bittencourt, L. F., Madeira, E. R., \& Villas, L. A.: Towards a distributed and infrastructure-less vehicular traffic management system. Computer Communications. (2020)

[47] Rajab, S.: Vehicle to Infrastructure Communications. In Connected Vehicles. 157-179 (2019) 
[48] Qayyum, A., Usama, M., Qadir, J., \& Al-Fuqaha, A.: Securing Future Autonomous \& Connected Vehicles: Challenges Posed by Adversarial Machine Learning and The Way Forward. IEEE Communications Surveys \& Tutorials. (2020)

[49] Eskandarian, A., Wu, C., \& Sun, C.: Research Advances and Challenges of Autonomous and Connected Ground Vehicles. (2019)

[50] Gikas, V., Retscher, G., \& Kealy, A.: Collaborative Positioning for Urban Intelligent Transportation Systems (ITS) and Personal Mobility (PM): Challenges and Perspectives. In Mobility Patterns, Big Data and Transport Analytics. 381-414 (2019)

[51] Wang, J., Shao, Y., Ge, Y., \& Yu, R.: A survey of vehicle to everything (v2x) testing. Sensors. 19(2), 334 (2019)

[52] Singh, P. K., Nandi, S. K., \& Nandi, S.: A tutorial survey on vehicular communication state of the art, and future research directions. Vehicular Communications. 18, 100164 (2019)

[53] Rozhnov, A. V., Lychev, A. V., \& Lobanov, I. A.: Hybrid Optimization Modeling Framework for Research Activities in Intelligent Data Processing. In Computer Vision in Control System. 6, 141-152 (2020)

[54] Farahani, S. S., Bleeker, C., van Wijk, A., \& Lukszo, Z.: Hydrogen-based integrated energy and mobility system for a real-life office environment. Applied Energy. 264, 114695 (2020)

[55] Noel, L., de Rubens, G. Z., Kester, J., \& Sovacool, B. K.: Vehicle-to-Grid: A Sociotechnical Transition Beyond Electric Mobility. (2019)

[56] Anjuman, T., Hasanat-E-Rabbi, S., Siddiqui, C. K. A., \& Hoque, M. M.: Road traffic accident: A leading cause of the global burden of public health injuries and fatalities. In Proc. Int. Conf. Mech. Eng. Dhaka Bangladesh. 29-31 (2020)

[57] Xin, C., Dan, L., \& Shuo, H.: Research on Deceleration Early Warning Model Based on V2X. In 2020 12th International Conference on Measuring Technology and Mechatronics Automation (ICMTMA). 318-321 (2020)

[58] Tan, J., Qin, X., \& Gong, L.: Using vehicle-to-vehicle communication to improve traffic safety in sand-dust environment. International journal of environmental research and public health. 17(4), 1165 (2020)

[59] Zhai, W. X., \& Ardian, D.: Traffic flow control of the intersection in urban traffic system under the environment of internet of vehicles. Advances in Transportation Studies. (2020)

[60] Masih-Tehrani, M., Ebrahimi-Nejad, S., \& Dahmardeh, M.: Combined fuel consumption and emission optimization model for heavy construction equipment. Automation in Construction. 110, 103007 (2020)

[61] Zhou, H., Xu, W., Chen, J., \& Wang, W.: Evolutionary V2X Technologies Toward the Internet of Vehicles: Challenges and Opportunities. Proceedings of the IEEE. 108(2), 308-323 (2020)

[62] Ameen, H. A., Mahamad, A. K., Saon, S., Nor, D. M., \& Ghazi, K.: A review on vehicle to vehicle communication system applications. Indonesian Journal of Electrical Engineering and Computer Science. 18(1), 188-198 (2020)

[63] Tripp-Barba, C., Zaldívar-Colado, A., Urquiza-Aguiar, L., \& Aguilar-Calderón, J. A.: Survey on Routing Protocols for Vehicular Ad Hoc Networks Based on Multimetrics. Electronics. 8(10), 1177 (2019)

[64] Darwish, T. S., Bakar, K. A., \& Haseeb, K.: Reliable intersection-based traffic aware routing protocol for urban areas vehicular ad hoc networks. IEEE Intelligent Transportation Systems Magazine. 10(1), 60-73 (2018)

[65] Srivastava, A., \& Prakash, A.: Reliable Location-Aware Routing Protocol for Urban Vehicular Scenario. In Advances in VLSI, Communication, and Signal Processing. 13-22 (2020)

[66] Vats, E. J., Tejpal, G., \& Sharma, S.: Routing Protocols and Attacks in Vehicular ADHOC Network (VANETs): A Review. International Journal. 6(12) (2016)

[67] Malnar, M., \& Jevtić, N.: A Framework for Performance Evaluation of VANETs Using NS-3 Simulator. PrometTraffic\&Transportation. 32(2), 255-268 (2020)

[68] Pandey, P. K., Swaroop, A., \& Kansal, V.: A Concise Survey on Recent Routing Protocols for Vehicular Ad hoc Networks (VANETs). In 2019 International Conference on Computing, Communication, and Intelligent Systems (ICCCIS). 188-193 (2019)

[69] Shin, J., Baek, Y., \& Son, S. H.: Fundamental topology-based routing protocols for autonomous vehicles. In 2016 IEEE 22nd International Conference on Embedded and Real-Time Computing Systems and Applications (RTCSA). 265-265 (2016)

[70] Srivastava, A., \& Prakash, A.: Reliable Location-Aware Routing Protocol for Urban Vehicular Scenario. In Advances in VLSI, Communication, and Signal Processing. 13-22 (2020)

[71] Ibrahim, B. F., Toycan, M., \& Mawlood, H. A.: A Comprehensive Survey on VANET Broadcast Protocols. In 2020 International Conference on Computation, Automation and Knowledge Management (ICCAKM). 298-302 (2020)

[72] Liu, J., Wan, J., Wang, Q., Deng, P., Zhou, K., \& Qiao, Y.: A survey on position-based routing for vehicular ad hoc networks. Telecommunication Systems. 62(1), 15-30 (2016)

[73] Vasudev, H., Das, D., \& Vasilakos, A. V.: Secure message propagation protocols for IoVs communication components. Computers \& Electrical Engineering. 82, 106555 (2020)

[74] Dhiman, A., \& Kumar, R.: A Comparative Study of Position Based Routing Protocols in VANETs. In 2019 International Conference on Intelligent Sustainable Systems (ICISS). 306-311 (2019)

[75] Abuashour, A., \& Kadoch, M.: Performance improvement of cluster-based routing protocol in VANET. IEEE Access. $5,15354-15371(2017)$

[76] Bengag, A., Bengag, A., \& Elboukhari, M.: Routing Protocols for VANETs: A Taxonomy, Evaluation and Analysis. Advances in Science, Technology and Engineering Systems Journal. 5(1), 77-85 (2020)

[77] Al Mushayt, O. S., Gharibi, W., \& Armi, N.: Multicast routing protocol for advanced vehicular ad hoc networks. Telkomnika. 17(3), 1096-1100 (2019)

[78] Verma, R., Rauthan, M. M. S., \& Vaisla, K. S.: A comparative analysis of multicast routing protocols in VANET for smart city scenario. In 2017 International Conference on Inventive Computing and Informatics (ICICI). 810-814 (2017) 
[79] Yeferny, T., \& Hamad, S.: Vehicular Ad-hoc Networks: Architecture, Applications and Challenges. IJCSNS. 20(2) (2020)

[80] Kumar, P., Verma, A., \& Singhal, P.: VANET Protocols with Challenges-A Review. In 2019 6th International Conference on Computing for Sustainable Global Development (INDIACom). 598-602 (2019)

[81] Devangavi, A. D., \& Gupta, R.: Routing protocols in VANET - a survey. In 2017 International Conference on Smart Technologies for Smart Nation (SmartTechCon). 163-167 (2017)

[82] Senouci, O., Aliouat, Z., \& Harous, S.: A review of routing protocols in internet of vehicles and their challenges. Sensor Review. (2019)

[83] Dutta, R., \& Thalore, R.: A Review of Various Routing Protocols in VANET. International Journal of Advanced Engineering Research and Science. 4(4) (2017)

[84] Patel, D., Faisal, M., Batavia, P., Makhija, S., \& Mani, M.: Overview of routing protocols in VANET. International Journal of Computer Applications. 136(9), 4-7 (2016)

[85] Haq, A., \& Faheem, Y.: A peer-to-peer communication based content distribution protocol for incentive-aware delay tolerant networks. Wireless Networks, 26(1), 583-601 (2020).

[86] Hassija, V., Chamola, V., Han, G., Rodrigues, J. J., \& Guizani, M. : Dagiov: A framework for vehicle to vehicle communication using directed acyclic graph and game theory. IEEE Transactions on Vehicular Technology, 69(4), 4182-4191 (2020).

[87] Hu, C. C.: Peer-to-Peer Data Dissemination for Deadline-Sensitive Streaming in VANETs. IEEE Access (2020).

[88] You, Z., Cheng, G., Wang, Y., Chen, P., \& Chen, S.: Cross-Layer and SDN Based Routing Scheme for P2P Communication in Vehicular Ad-Hoc Networks. Applied Sciences, 9(22), 4734. (2019).

[89] Yang, L., Mo, T., \& Li, H.: Research on V2V Communication Based on Peer to Peer Network. In 2018 International Conference on Intelligent Autonomous Systems (ICoIAS) (pp. 105-110). IEEE (2018).

[90] Beak, B., Zamanipour, M., Head, K. L., \& Leonard, B.: Peer-to-peer priority signal control strategy in a connected vehicle environment. Transportation Research Record. 2672(18), 15-26 (2018).

[91] Malekian, R., Thakur, A., Nair, L., \& Pedersen, C. F.: A Sensor Based Peer to Peer Vehicle Data Sharing System, An Internet of Vehicles Approach. Journal of Internet Technology, 19(7), 2155-2162 (2018).

[92] Ferrag, M. A., \& Ahmim, A.: ESSPR: an efficient secure routing scheme based on searchable encryption with vehicle proxy re-encryption for vehicular peer-to-peer social network. Telecommunication Systems, 66(3), 481-503 (2017).

[93] Meneguette, R. I., \& Boukerche, A.: Peer-to-peer protocol for allocated resources in vehicular cloud based on V2V communication. In 2017 IEEE Wireless Communications and Networking Conference (WCNC) 1-6 (2017).

[94] Eshteiwi, K., Fredj, K. B., Kaddoum, G., \& Gagnon, F.: Performance analysis of peer-to-peer V2V wireless communications in the presence of interference. In 2017 IEEE 28th Annual International Symposium on Personal, Indoor, and Mobile Radio Communications (PIMRC). 1-6 (2017). 